\title{
Extended local convergence for Newton-type solver under weak conditions
}

\author{
Ioannis K. Argyros, Santhosh George and Kedarnath Senapati
}

\begin{abstract}
We present the local convergence of a Newton-type solver for equations involving Banach space valued operators. The eighth order of convergence was shown earlier in the special case of the $k$-dimensional Euclidean space, using hypotheses up to the eighth derivative although these derivatives do not appear in the method. We show convergence using only the first derivative. This way we extend the applicability of the methods. Numerical examples are used to show the convergence conditions. Finally, the basins of attraction of the method, on some test problems are presented.
\end{abstract}

Mathematics Subject Classification (2010): 65F08, 37F50, 65N12.

Keywords: Banach space, Newton-type, local convergence, Fréchet derivative.

\section{Introduction}

Let $\Omega \subset \mathcal{B}_{1}$ be nonempty, open, and $\mathcal{B}_{1}, \mathcal{B}_{2}$ be Banach spaces.

$$
\begin{gathered}
\mathcal{B}\left(\mathcal{B}_{1}, \mathcal{B}_{2}\right)=\left\{G: \mathcal{B}_{1} \longrightarrow \mathcal{B}_{2} \text { be bounded and linear }\right\}, \\
T(x, d)=\left\{y \in \mathcal{B}_{1}:\|y-x\|<d ; d>0\right\}
\end{gathered}
$$

and

$$
\bar{T}(x, d)=\left\{y \in \mathcal{B}_{1}:\|y-x\| \leq d ; d>0\right\} .
$$

One of the greatest challenges in Computational Mathematics is to find a solution $x_{*}$ of the equation

$$
\mathcal{F}(x)=0,
$$

where $\mathcal{F}: \Omega \longrightarrow \mathcal{B}_{2}$ is Fréchet differentiable operator. Notice that a plethora of applications from Mathematics, Science and Engineering are reduced to a form as (1.1) by utilizing Mathematical modeling [1-19]. The solution $x_{*}$ is sought in closed form, but this can be achieved only in some cases. Hence, researchers develop iterative methods, generating a sequence approximating $x_{*}$ under certain initial conditions. 
Newton's is clearly the most popular method converging quadratically to $x_{*}$, and given as

$$
x_{n+1}=x_{n}-\mathcal{F}^{\prime}\left(x_{n}\right)^{-1} \mathcal{F}(x) \text { for all } n=0,1,2, \ldots,
$$

with $x_{0} \in \Omega$. Chebyshev, Halley methods have been studied extensively which are of order three but use the expensive $\mathcal{F}^{\prime \prime}$ at each step as well as $\mathcal{F}^{\prime}\left(x_{n}\right)^{-1}$. That is why researchers have introduced methods using divided differences of order one such as Secant, Steffensen, Kurchatov, and Aitken methods [2, 3, 4, 10, 13, 14].

In particular, we are concerned with the local convergence of the Newton-type method given as

$$
\begin{aligned}
x_{0} \in \Omega, y_{n} & =x_{n}-\mathcal{F}^{\prime}\left(x_{n}\right)^{-1} \mathcal{F}\left(x_{n}\right) \\
z_{n} & =y_{n}-\left[\frac{13}{4} I-Q\left(x_{n}\right)\left(\frac{7}{2} I-\frac{5}{4} Q\left(x_{n}\right)\right)\right] \mathcal{F}^{\prime}\left(x_{n}\right)^{-1} \mathcal{F}\left(y_{n}\right) \\
x_{n+1} & =z_{n}-\left[\frac{7}{2} I-Q\left(x_{n}\right)\left(4 I-\frac{3}{2} Q\left(x_{n}\right)\right)\right] \mathcal{F}^{\prime}\left(x_{n}\right)^{-1} \mathcal{F}\left(z_{n}\right),
\end{aligned}
$$

where $Q\left(x_{n}\right)=\mathcal{F}^{\prime}\left(x_{n}\right)^{-1} \mathcal{F}\left(y_{n}\right)$. Method (1.3) was studied in [17], but for the case $\mathcal{B}_{1}=\mathcal{B}_{2}=\mathbb{R}^{k}$ ( $k$ a natural number). The convergence order was established by conditions on high order derivative, and Taylor series, although these derivatives do not appear in the method (1.3). Therefore, these hypotheses limit the usage of the method (1.3).

As an academic example: Let $\mathcal{B}_{1}=\mathcal{B}_{2}=\mathbb{R}, \Omega=\left[-\frac{1}{2}, \frac{3}{2}\right]$. Define $\mathcal{F}$ on $\Omega$ by

$$
\mathcal{F}(x)=x^{3} \log x^{2}+x^{5}-x^{4}
$$

Then, we have $x_{*}=1$, and

$$
\begin{gathered}
\mathcal{F}^{\prime}(x)=3 x^{2} \log x^{2}+5 x^{4}-4 x^{3}+2 x^{2}, \\
\mathcal{F}^{\prime \prime}(x)=6 x \log x^{2}+20 x^{3}-12 x^{2}+10 x, \\
\mathcal{F}^{\prime \prime \prime}(x)=6 \log x^{2}+60 x^{2}-24 x+22 .
\end{gathered}
$$

Obviously $\mathcal{F}^{\prime \prime \prime}(x)$ is not bounded on $\Omega$. So, the convergence of method (1.3) not guaranteed by the analysis in [15], [17], [18].

Other problems with the usage of the method (1.3) are: no information on how to choose the initial point $x_{0}$; bounds on $\left\|x_{n}-x_{*}\right\|$ and information on the location of $x_{*}$. All these are addressed in this paper by only using conditions on the first derivative, and in the more general setting of Banach space valued operators. That is how, we expand the applicability of the method (1.3). To avoid the usage of Taylor series and high convergence order derivatives, we rely on the computational order of convergence (COC) or the approximate computational order of convergence (ACOC) $[2,3,4]$.

The layout of the rest of the paper includes: the local convergence analysis in Section 2, some numerical examples in Section 3 and the basins of attraction in Section 4 . 


\section{Local convergence analysis}

We shall introduce some scalar functions and parameters that appear in the local convergence analysis of the method (1.3). Let $\varphi_{0}:[0, \infty) \longrightarrow[0, \infty)$ be an increasing and continuous function satisfying $\varphi_{0}(0)=0$.

Suppose that the equation

$$
\varphi_{0}(t)=1
$$

has at least one positive solution. Denote by $\rho_{0}$ the smallest such solution. Let $\varphi$ : $\left[0, \rho_{0}\right) \longrightarrow[0, \infty)$ and $\varphi_{1}:\left[0, \rho_{0}\right) \longrightarrow[0, \infty)$ be increasing and continuous functions with $\varphi(0)=0$. Define functions $\psi_{1}$ and $\bar{\psi}_{1}$ on the interval $\left[0, \rho_{0}\right)$ by

$$
\psi_{1}(t)=\frac{\int_{0}^{1} \varphi((1-\theta) t) d \theta}{1-\varphi_{0}(t)}
$$

and

$$
\bar{\psi}_{1}(t)=\psi_{1}(t)-1
$$

We have $\bar{\psi}_{1}(0)=-1$ and $\bar{\psi}_{1}(t) \longrightarrow \infty$ as $t \longrightarrow \rho_{0}^{-}$. The intermediate value theorem assures that the equation $\bar{\psi}_{1}(t)=0$ has at least one solution in $\left(0, \rho_{0}\right)$. Denote by $r_{1}$ the smallest such solution.

Suppose that the equation

$$
\varphi_{0}\left(\psi_{1}(t) t\right)=1
$$

has at least one positive solution. Denote by $\rho_{1}$ the smallest such solution.

Set $\rho_{2}=\min \left\{\rho_{0}, \rho_{1}\right\}$. Define the functions $\psi_{2}$ and $\bar{\psi}_{2}$ on $\left[0, \rho_{2}\right)$ by

$$
\begin{aligned}
\psi_{2}(t)= & \left\{\frac{\int_{0}^{1} \varphi\left((1-\theta) \psi_{1}(t) t\right) d \theta}{1-\varphi_{0}\left(\psi_{1}(t) t\right)}\right. \\
& +\frac{\left(\varphi_{0}\left(\psi_{1}(t) t\right)+\varphi_{0}(t)\right) \int_{0}^{1} \varphi_{1}\left(\theta \psi_{1}(t) t\right) d \theta}{\left(1-\varphi_{0}\left(\psi_{1}(t) t\right)\right)\left(1-\varphi_{0}(t)\right)} \\
& +\frac{1}{4}\left[\frac{9\left(\varphi_{0}\left(\psi_{1}(t) t\right)+\varphi_{0}(t)\right)}{1-\varphi_{0}(t)}\right] \\
& \left.+\frac{5 \varphi_{1}\left(\psi_{1}(t) t\right)\left(\varphi_{0}\left(\psi_{1}(t) t\right)+\varphi_{0}(t)\right)}{\left(1-\varphi_{0}(t)\right)^{2}}\right] \\
& \left.\times \frac{\int_{0}^{1} \varphi_{1}\left(\theta \psi_{1}(t) t\right) d \theta}{1-\varphi_{0}(t)}\right\} \psi_{1}(t),
\end{aligned}
$$

and $\bar{\psi}_{2}(t)=\psi_{2}(t)-1$. We get $\bar{\psi}_{2}(0)=-1$ and $\bar{\psi}_{2}(t) \longrightarrow \infty$ as $t \longrightarrow \rho_{2}^{-}$. Denote by $r_{2}$ the smallest solution of equation $\bar{\psi}_{2}(t)=0$ in $\left(0, \rho_{2}\right)$.

Suppose that the equation

$$
\varphi_{0}\left(\psi_{2}(t) t\right)=1
$$


has at least one positive solution. Denote by $\rho_{3}$ the smallest such solution. Set $\rho=\min \left\{\rho_{2}, \rho_{3}\right\}$. Define the functions $\psi_{3}$ and $\bar{\psi}_{3}$ on the interval $[0, \rho)$ by

$$
\begin{aligned}
\psi_{3}(t)= & \left\{\frac{\int_{0}^{1} \varphi\left((1-\theta) \psi_{2}(t) t\right) d \theta}{1-\varphi_{0}\left(\psi_{2}(t) t\right)}\right. \\
& +\frac{\left(\varphi_{0}\left(\psi_{2}(t) t\right)+\varphi_{0}(t)\right) \int_{0}^{1} \varphi_{1}\left(\theta \psi_{2}(t) t\right) d \theta}{\left(1-\varphi_{0}\left(\psi_{2}(t) t\right)\right)\left(1-\varphi_{0}(t)\right)} \\
& +\frac{1}{2}\left[\frac{5\left(\varphi_{0}\left(\psi_{2}(t) t\right)+\varphi_{0}(t)\right)}{1-\varphi_{0}(t)}\right] \\
& \left.+\frac{3\left(\varphi_{1}\left(\psi_{1}(t) t\right)\left(\varphi_{0}\left(\psi_{2}(t) t\right)+\varphi_{0}(t)\right)\right.}{\left(1-\varphi_{0}(t)\right)^{2}}\right] \\
& \left.\times \frac{\int_{0}^{1} \varphi_{1}\left(\theta \psi_{2}(t) t\right) d \theta}{1-\varphi_{0}(t)}\right\} \psi_{2}(t)
\end{aligned}
$$

and $\bar{\psi}_{3}(t)=\psi_{3}(t)-1$. We get $\bar{\psi}_{3}(0)=-1$ and $\bar{\psi}_{3}(t) \longrightarrow \infty$ as $t \longrightarrow \rho^{-}$. Denote by $r_{3}$ the smallest solution of the equation $\bar{\psi}_{3}(t)=0$ in $(0, \rho)$. Define a radius of convergence $r$ by

$$
r=\min \left\{r_{i}\right\}, i=1,2,3 .
$$

It follows that for each $t \in[0, r)$

$$
\begin{aligned}
& 0 \leq \varphi_{0}(t)<1 \\
& 0 \leq \varphi_{0}\left(\psi_{1}(t) t\right)<1 \\
& 0 \leq \varphi_{0}\left(\psi_{2}(t) t\right)<1
\end{aligned}
$$

and

$$
0 \leq \psi_{i}(t)<1, i=1,2,3 \text {. }
$$

The local convergence analysis of the method (1.3) use the hypotheses $(\mathrm{H})$ :

(h1) $\mathcal{F}: \Omega \longrightarrow \mathcal{B}_{2}$ a continuously differentiable operator in the sense of Fréchet and there exists $x_{*} \in \Omega$ such that $\mathcal{F}\left(x_{*}\right)=0$, and $\mathcal{F}^{\prime}\left(x_{*}\right)^{-1} \in \mathcal{B}\left(\mathcal{B}_{2}, \mathcal{B}_{1}\right)$.

(h2) There exists function $\varphi_{0}:[0, \infty) \longrightarrow[0, \infty)$ continuous, and increasing with $\varphi_{0}(0)=0$ such that for each $x \in \Omega$

$$
\left\|\mathcal{F}^{\prime}\left(x_{*}\right)^{-1}\left(F^{\prime}(x)-F^{\prime}\left(x_{*}\right)\right)\right\| \leq \varphi_{0}\left(\left\|x-x_{*}\right\|\right)
$$

and $\rho_{0}$ given by $(2.1)$ exists. Set $\Omega_{0}=\Omega \cap T\left(x_{*}, \rho_{0}\right)$.

(h3) There exist functions $\varphi:\left[0, \rho_{0}\right) \longrightarrow[0, \infty), \varphi_{1}:\left[0, \rho_{0}\right) \longrightarrow[0, \infty)$ continuous, and increasing such that for each $x, y \in \Omega_{0}$

$$
\left.\| \mathcal{F}^{\prime}\left(x_{*}\right)^{-1}(\mathcal{F}(y)]-\mathcal{F}^{\prime}(x)\right) \| \leq \varphi(\|y-x\|
$$

and

$$
\left.\| \mathcal{F}^{\prime}\left(x_{*}\right)^{-1} \mathcal{F}^{\prime}(x)\right) \| \leq \varphi_{1}\left(\left\|x-x_{*}\right\| .\right.
$$

(h4) $\bar{T}\left(x_{*}, r\right) \subseteq \Omega$ and $\rho_{1}, \rho_{2}$ exist and are given by (2.2), (2.3), respectively and $r$ is defined in (2.4).

(h5) There exists $r_{*} \geq r$ such that $\int_{0}^{1} \varphi_{0}\left(\theta r_{*}\right) d \theta<1$.

Set $\Omega_{1}=\Omega \cap \bar{T}\left(x_{*}, r_{*}\right)$. 
Next, we present the local convergence analysis of the method (1.3) using preceding notation and the hypotheses $(\mathrm{H})$.

Theorem 2.1. Suppose that the hypotheses (H) hold, and choose $x_{0} \in T\left(x_{*}, r_{*}\right)-\left\{x_{*}\right\}$. Then, the sequence $\left\{x_{n}\right\}$ starting at $x_{0}$ and generated by the method (1.3) is well defined, remains in $T\left(x_{*}, r\right)$ for each $n=0,1,2, \ldots$ and converges to $x_{*}$. Moreover the following error bounds hold

$$
\begin{gathered}
\left\|y_{n}-x_{*}\right\| \leq \psi_{1}\left(\left\|x_{n}-x_{*}\right\|\right)\left\|x_{n}-x_{*}\right\| \leq\left\|x_{n}-x_{*}\right\|<r, \\
\left\|z_{n}-x_{*}\right\| \leq \psi_{2}\left(\left\|x_{n}-x_{*}\right\|\right)\left\|x_{n}-x_{*}\right\| \leq\left\|x_{n}-x_{*}\right\|, \\
\left\|x_{n+1}-x_{*}\right\| \leq \psi_{3}\left(\left\|x_{n}-x_{*}\right\|\right)\left\|x_{n}-x_{*}\right\| \leq\left\|x_{n}-x_{*}\right\|,
\end{gathered}
$$

where functions $\psi_{i}$ are given previously and $r$ is defined in (2.4). Furthermore, the limit point $x_{*}$ is the only solution of equation $\mathcal{F}(x)=0$ in the set $\Omega_{1}$ given in (h5).

Proof. Estimates (2.9)-(2.11) shall be shown using mathematical induction. Let

$$
x \in T\left(x_{*}, r\right)-\left\{x_{*}\right\} .
$$

By (2.4), (h1) and (h2), we have in turn that

$$
\left\|\mathcal{F}^{\prime}\left(x_{*}\right)^{-1}\left(\mathcal{F}^{\prime}(x)-\mathcal{F}^{\prime}\left(x_{*}\right)\right)\right\| \leq \varphi_{0}\left(\left\|x-x_{*}\right\|\right)<\varphi_{0}(r)<1 .
$$

Estimate (2.12) and the Banach lemma on invertible operators [2, 14] assure that $\mathcal{F}^{\prime}(x)^{-1} \in \mathcal{B}\left(\mathcal{B}_{2}, \mathcal{B}_{1}\right)$ and

$$
\left\|\mathcal{F}^{\prime}(x)^{-1} \mathcal{F}^{\prime}\left(x_{*}\right)\right\| \leq \frac{1}{1-\varphi_{0}\left(\left\|x-x_{*}\right\|\right)} .
$$

It also follows that, for $x=x_{0}$, iterates $y_{0}, z_{0}, x_{1}$ are well defined by the method (1.3) for $n=0$. We get from the first substep of the method (1.3) for $n=0$ and (h1) that

$$
y_{0}-x_{*}=x_{0}-x_{*}-\mathcal{F}^{\prime}\left(x_{0}\right)^{-1} \mathcal{F}\left(x_{0}\right),
$$

so by $(2.4),(2.8)$ (for $i=1),($ h3), (2.13) and (2.14), we obtain in turn that

$$
\begin{aligned}
\left\|y_{0}-x_{*}\right\| \leq & \left\|\mathcal{F}^{\prime}\left(x_{0}\right)^{-1} \mathcal{F}^{\prime}\left(x_{*}\right)\right\| \\
& \times\left\|\int_{0}^{1} \mathcal{F}^{\prime}\left(x_{*}\right)^{-1}\left(\mathcal{F}^{\prime}\left(x_{*}+\theta\left(x_{0}-x_{*}\right)\right)-\mathcal{F}^{\prime}\left(x_{0}\right)\right)\left(x_{0}-x_{*}\right) d \theta\right\| \\
\leq & \frac{\int_{0}^{1} \varphi\left((1-\theta)\left\|x_{0}-x_{*}\right\|\right) d \theta\left\|x_{0}-x_{*}\right\|}{1-\varphi_{0}\left(\left\|x_{0}-x_{*}\right\|\right)} \\
= & \psi_{1}\left(\left\|x_{0}-x_{*}\right\|\left\|x_{0}-x_{*}\right\| \leq\left\|x_{0}-x_{*}\right\|<r\right.
\end{aligned}
$$

which shows $(2.9)$ for $n=0$ and $y_{0} \in T\left(x_{*}, r\right)$. The second substep of the method (1.3) can be written as

$$
\begin{aligned}
z_{0}-x_{*}= & \left(y_{0}-x_{*}-\mathcal{F}^{\prime}\left(y_{0}\right)^{-1} \mathcal{F}\left(y_{0}\right)\right)+\left(\mathcal{F}^{\prime}\left(y_{0}\right)^{-1}-\mathcal{F}^{\prime}\left(x_{0}\right)^{-1}\right) \mathcal{F}\left(y_{0}\right) \\
& -\frac{1}{4}\left[9\left(I-Q\left(x_{0}\right)\right)-5 Q\left(x_{0}\right)\left(I-Q\left(x_{0}\right)\right)\right] Q\left(x_{0}\right) .
\end{aligned}
$$


Then, by (2.4), (2.8) (for $i=2$ ), (2.13) (for $i=2$ ), (2.15) and (2.16), we get in turn that

$$
\begin{aligned}
\left\|z_{0}-x_{*}\right\|= & \left\|y_{0}-x_{*}-\mathcal{F}^{\prime}\left(y_{0}\right)^{-1} \mathcal{F}\left(y_{0}\right)\right\| \\
& +\left\|\mathcal{F}^{\prime}\left(y_{0}\right)^{-1} \mathcal{F}^{\prime}\left(x_{*}\right)\right\|\left[\left\|\mathcal{F}^{\prime}\left(x_{*}\right)^{-1}\left(\mathcal{F}^{\prime}\left(y_{0}\right)-\mathcal{F}^{\prime}\left(x_{*}\right)\right)\right\|\right. \\
& \left.+\left\|\mathcal{F}^{\prime}\left(x_{*}\right)^{-1}\left(\mathcal{F}^{\prime}\left(x_{0}\right)-\mathcal{F}^{\prime}\left(x_{*}\right)\right)\right\|\right] \\
& +\frac{1}{4}\left[9\left\|I-Q\left(x_{0}\right)\right\|+5\left\|Q\left(x_{0}\right)\right\|\left\|I-Q\left(x_{0}\right)\right\|\right]\left\|Q\left(x_{0}\right)\right\| \\
\leq \quad & \left\{\frac{\int_{0}^{1} \varphi\left((1-\theta)\left\|y_{0}-x_{*}\right\|\right) d \theta}{1-\varphi_{0}\left(\left\|y_{0}-x_{*}\right\|\right)}\right. \\
& +\frac{\left(\varphi_{0}\left(\left\|y_{0}-x_{*}\right\|\right)+\varphi_{0}\left(\left\|x_{0}-x_{*}\right\|\right)\right) \int_{0}^{1} \varphi_{1}\left(\theta\left\|y_{0}-x_{*}\right\|\right) d \theta}{\left(1-\varphi_{0}\left(\left\|y_{0}-x_{*}\right\|\right)\left(1-\varphi_{0}\left(\left\|x_{0}-x_{*}\right\|\right)\right)\right.} \\
& +\frac{1}{4}\left[\frac{9\left(\varphi_{0}\left(\left\|x_{0}-x_{*}\right\|\right)+\varphi_{0}\left(\left\|y_{0}-x_{*}\right\|\right)\right)}{1-\varphi_{0}\left(\left\|x_{0}-x_{*}\right\|\right)}\right. \\
& \left.+\frac{5 \varphi_{1}\left(\left\|y_{0}-x_{*}\right\|\right)\left(\varphi_{0}\left(\left\|x_{0}-x_{*}\right\|\right)+\varphi_{0}\left(\left\|y_{0}-x_{*}\right\|\right)\right)}{\left(1-\varphi_{0}\left(\left\|x_{0}-x_{*}\right\|\right)\right)^{2}}\right] \\
& \left.\times \frac{\int_{0}^{1} \varphi_{1}\left(\theta\left\|y_{0}-x_{*}\right\|\right) d \theta}{1-\varphi_{0}\left(\left\|x_{0}-x_{*}\right\|\right)}\right\}\left\|y_{0}-x_{*}\right\| \\
\leq \quad & \psi_{2}\left(\left\|x_{0}-x_{*}\right\|\right)\left\|x_{0}-x_{*}\right\| \leq\left\|x_{0}-x_{*}\right\|<r,
\end{aligned}
$$

which shows $(2.12)$ for $n=0$, and $z_{0} \in T\left(x_{*}, r\right)$. Using the third substep of the method (1.3) for $n=0$, we can write

$$
\begin{aligned}
x_{1}-x_{*}= & \left(z_{0}-x_{*}-\mathcal{F}^{\prime}\left(z_{0}\right)^{-1} \mathcal{F}\left(z_{0}\right)\right)+\left(\mathcal{F}^{\prime}\left(z_{0}\right)^{-1}-\mathcal{F}^{\prime}\left(x_{0}\right)^{-1}\right) \mathcal{F}\left(z_{0}\right) \\
& -\left[\frac{5}{2} I-Q\left(x_{0}\right)\left(4 I-\frac{3}{2} Q\left(x_{0}\right)\right)\right] \mathcal{F}^{\prime}\left(x_{0}\right)^{-1} \mathcal{F}\left(z_{0}\right) \\
= & \left(z_{0}-x_{*}-\mathcal{F}^{\prime}\left(z_{0}\right)^{-1} \mathcal{F}\left(z_{0}\right)\right) \\
& \mathcal{F}^{\prime}\left(z_{0}\right)^{-1}\left[\left(\mathcal{F}^{\prime}\left(x_{0}\right)-\mathcal{F}^{\prime}\left(x_{*}\right)\right)+\left(\mathcal{F}^{\prime}\left(x_{*}\right)-\mathcal{F}^{\prime}\left(z_{0}\right)\right)\right] F\left(z_{0}\right) \\
& -\frac{1}{2}\left[5\left(I-Q\left(x_{0}\right)\right)-3 Q\left(x_{0}\right)\left(I-Q\left(x_{0}\right)\right)\right] \mathcal{F}^{\prime}\left(x_{0}\right)^{-1} \mathcal{F}\left(z_{0}\right) .
\end{aligned}
$$

Then, using (2.4), (2.8) (for $i=3$ ), (2.13) (for $\left.x=z_{0}\right),(2.15),(2.17)$ and (2.18), we have in turn as in (2.17) that

$$
\begin{aligned}
\left\|x_{1}-x_{*}\right\| \leq & \left\{\frac{\int_{0}^{1} \varphi\left((1-\theta)\left\|z_{0}-x_{*}\right\|\right) d \theta}{1-\varphi_{0}\left(\left\|z_{0}-x_{*}\right\|\right)}\right. \\
& +\frac{\left(\varphi_{0}\left(\left\|z_{0}-x_{*}\right\|\right)+\varphi_{0}\left(\left\|x_{0}-x_{*}\right\|\right)\right) \int_{0}^{1} \varphi_{1}\left(\theta\left\|z_{0}-x_{*}\right\|\right) d \theta}{\left(1-\varphi_{0}\left(\left\|z_{0}-x_{*}\right\|\right)\left(1-\varphi_{0}\left(\left\|x_{0}-x_{*}\right\|\right)\right)\right.}
\end{aligned}
$$




$$
\begin{aligned}
& +\frac{1}{2}\left[\frac{5\left(\varphi_{0}\left(\left\|x_{0}-x_{*}\right\|\right)+\varphi_{0}\left(\left\|y_{0}-x_{*}\right\|\right)\right)}{1-\varphi_{0}\left(\left\|x_{0}-x_{*}\right\|\right)}\right. \\
& \left.\frac{3 \varphi_{1}\left(\left\|y_{0}-x_{*}\right\|\right)\left(\varphi_{0}\left(\left\|x_{0}-x_{*}\right\|\right)+\varphi_{0}\left(\left\|y_{0}-x_{*}\right\|\right)\right)}{\left(1-\varphi_{0}\left(\left\|x_{0}-x_{*}\right\|\right)\right)^{2}}\right] \\
& \left.\quad \times \frac{\int_{0}^{1} \varphi_{1}\left(\theta\left\|z_{0}-x_{*}\right\|\right) d \theta}{1-\varphi_{0}\left(\left\|x_{0}-x_{*}\right\|\right)}\right\}\left\|z_{0}-x_{*}\right\| \\
& \leq \quad \psi_{3}\left(\left\|x_{0}-x_{*}\right\|\right)\left\|x_{0}-x_{*}\right\| \leq\left\|x_{0}-x_{*}\right\|<r,
\end{aligned}
$$

which shows (2.12) for $n=0$ and $x_{1} \in T\left(x_{*}, r\right)$. The induction for (2.9)-(2.12) is finished, by simply replacing $x_{0}, y_{0}, z_{0}, x_{1}$ by $x_{m}, y_{m}, z_{m}, x_{m+1}$ in the preceding estimates. Then, using the estimate

$$
\left\|x_{m+1}-x_{*}\right\| \leq c\left\|x_{m}-x_{*}\right\| \leq\left\|x_{m}-x_{*}\right\|<r,
$$

where $c=\psi_{3}\left(\left\|x_{0}-x_{*}\right\|\right) \in[0,1)$, we deduce that $x_{m+1} \in T\left(x_{*}, r\right)$, and

$$
\lim _{m \longrightarrow \infty} x_{m}=x_{*} \text {. }
$$

Finally, to show the uniqueness part, let $y_{*} \in \Omega_{1}$ with $\mathcal{F}\left(y_{*}\right)=0$. Define

$$
G=\int_{0}^{1} \mathcal{F}^{\prime}\left(x_{*}+\theta\left(y_{*}-x_{*}\right)\right) d \theta .
$$

Then, using (h2) and (h5), we get in turn that

$$
\left\|\mathcal{F}^{\prime}\left(p_{*}\right)^{-1}\left(G-\mathcal{F}^{\prime}\left(x_{*}\right)\right)\right\| \leq \int_{0}^{1} \varphi_{0}\left(\theta\left\|y_{*}-x_{*}\right\|\right) d \theta \leq \int_{0}^{1} \varphi_{0}\left(\theta r_{*}\right) d \theta<1,
$$

so $G^{-1}$ exists, and from

$$
0=\mathcal{F}\left(x_{*}\right)-\mathcal{F}\left(y_{*}\right)=G\left(x_{*}-y_{*}\right)
$$

we derive $x_{*}=y_{*}$.

Remark 2.2. (a) In the case when $\varphi_{0}(t)=L_{0} t, \varphi(t)=L t$ and $\Omega_{0}=\Omega$, the radius

$$
\rho_{A}=\frac{2}{2 L_{0}+L}
$$

was obtained by Argyros et al. in [4] as the convergence radius for Newton's method under condition (2.7)-(2.9). Notice that the convergence radius for Newton's method, given independently by Rheinboldt [15] and Traub [19] is given by

$$
\rho_{T R}=\frac{2}{3 L}<\rho_{A} .
$$

As an example, let us consider the function $F(x)=e^{x}-1$. Then $\alpha^{*}=0$. Set

$$
\Omega=B(0,1) .
$$

Then, we have that $L_{0}=e-1<L=e$, so

$$
\rho_{T R}=0.24252961<\rho_{A}=0.324947231 .
$$


(b) The local results can be used for projection methods such as Arnoldi's method, the generalized minimum residual method (GMREM), the generalized conjugate method (GCM) for combined Newton/finite projection methods and in connection to the mesh independence principle in order to develop the cheapest and most efficient mesh refinement strategy $[2,3,4,10]$.

(c) The results can also be used to solve equations where the operator $F^{\prime}$ satisfies the autonomous differential equation $[2,3,4,10]$ :

$$
F^{\prime}(x)=P(F(x))
$$

where $P: \mathcal{B}_{2} \longrightarrow \mathcal{B}_{2}$ is a known continuous operator. Since

$$
F^{\prime}\left(x^{*}\right)=P\left(F\left(x^{*}\right)\right)=P(0),
$$

we can apply the results without actually knowing the solution $x^{*}$. Consider as an example $F(x)=e^{x}-1$. Then, we can choose $P(x)=x+1$ and $x^{*}=0$.

(d) It is worth noticing that the method (1.3) does not change when we use the conditions of the preceding Theorem instead of the stronger conditions used in [17]. Moreover, we can compute the computational order of convergence (COC) which is defined as

$$
\xi=\ln \left(\frac{\left\|x_{n+1}-x_{*}\right\|}{\left\|x_{n}-x_{*}\right\|}\right) / \ln \left(\frac{\left\|x_{n}-x_{*}\right\|}{\left\|x_{n-1}-x_{*}\right\|}\right)
$$

or the approximate computational order of convergence (ACOC)

$$
\xi_{1}=\ln \left(\frac{\left\|x_{n+1}-x_{n}\right\|}{\left\|x_{n}-x_{n-1}\right\|}\right) / \ln \left(\frac{\left\|x_{n}-x_{n-1}\right\|}{\left\|x_{n-1}-x_{n-2}\right\|}\right) .
$$

This way we obtain in practice, the order of convergence, in which higher order derivatives are not used.

\section{Numerical example}

We present the following example to test the convergence criteria.

Example 3.1. Let $\mathcal{B}_{1}=\mathcal{B}_{1}=\mathbb{R}^{3}, \Omega=U(0,1), x_{*}=(0,0,0)^{T}$ and define $\mathcal{F}$ on $\Omega$ by

$$
\mathcal{F}(x)=\mathcal{F}\left(u_{1}, u_{2}, u_{3}\right)=\left(e^{u_{1}}-1, \frac{e-1}{2} u_{2}^{2}+u_{2}, u_{3}\right)^{T} .
$$

For the points $u=\left(u_{1}, u_{2}, u_{3}\right)^{T}$, the Fréchet derivative is given by

$$
\mathcal{F}^{\prime}(u)=\left(\begin{array}{ccc}
e^{u_{1}} & 0 & 0 \\
0 & (e-1) u_{2}+1 & 0 \\
0 & 0 & 1
\end{array}\right)
$$

Using the norm of the maximum of the rows $x_{*}=(0,0,0)^{T}$ and since

$$
\mathcal{F}^{\prime}\left(x_{*}\right)=\operatorname{diag}(1,1,1),
$$


we get by conditions $(\mathrm{H}), \varphi_{0}(t)=(e-1) t, \varphi(t)=e^{\frac{1}{e-1}} t, \varphi_{1}(t)=e^{\frac{1}{e-1}}$, and

$$
\begin{aligned}
& r_{1}=0.3826919122323857447, \\
& r_{2}=0.127735710261785623265, \\
& r_{3}=0.089354652353140273657=r .
\end{aligned}
$$

Example 3.2. Let $\mathcal{B}_{1}=\mathcal{B}_{2}=C[0,1], \Omega=\bar{U}(0,1)$. Define function $F$ on $\Omega$ by

$$
F(\varphi)(x)=\varphi(x)-5 \int_{0}^{1} x \theta \varphi(\theta)^{3} d \theta
$$

Then, the Fréchet-derivative is given by

$$
F^{\prime}(\varphi(\xi))(x)=\xi(x)-15 \int_{0}^{1} x \theta \varphi(\theta)^{2} \xi(\theta) d \theta, \text { for each } \xi \in \Omega .
$$

Then, we have that $x^{*}=0, \varphi_{0}(t)=L_{0} t, \varphi(t)=L t, \varphi_{1}(t)=2, L_{0}=7.5<L=15$. Then, the radius of convergence are given by

$$
\begin{aligned}
& r_{1}=0.0666666666666666666, \\
& r_{2}=0.0112691816233373796191, \\
& r_{3}=0.005991340191821196460575=r .
\end{aligned}
$$

Example 3.3. Returning to the motivational example given in the introduction of this study, we can choose $\varphi_{0}(t)=\varphi(t)=97$ and $\varphi_{1}(t)=1+\varphi_{0}(t)$. Then, the radius of convergence are given by

$$
\begin{aligned}
& r_{1}=0.0068728522336769759, \\
& r_{2}=0.0005865188569803861, \\
& r_{3}=1=0.000189538690198228865=r .
\end{aligned}
$$

\section{Basins of attraction}

As in [12] (also see references in [9]), we analyse the basins of attraction of the method (1.3). Recall that the basins of attraction of an iterative method are the collection of all initial points from which the iterative method converges to a solution of an equation [9]. The following test problems which are systems of polynomials in two variables are considered.

Example 4.1. $\left\{\begin{array}{l}x^{3}-y=0 \\ y^{3}-x=0\end{array}\right.$

with solutions $\{(-1,-1),(0,0),(1,1)\}$.

Example 4.2. $\left\{\begin{array}{l}3 x^{2} y-y^{3}=0 \\ x^{3}-3 x y^{2}-1=0\end{array}\right.$

with solutions $\left\{\left(-\frac{1}{2},-\frac{\sqrt{3}}{2}\right),\left(-\frac{1}{2}, \frac{\sqrt{3}}{2}\right),(1,0)\right\}$.

Example 4.3. $\left\{\begin{array}{l}x^{2}+y^{2}-4=0 \\ 3 x^{2}+7 y^{2}-16=0\end{array}\right.$

with solutions $\{(\sqrt{3}, 1),(-\sqrt{3}, 1),(\sqrt{3},-1),(-\sqrt{3},-1)\}$. 
For generating basins of attraction associated with roots of system of nonlinear equations, we consider a rectangular domain

$$
\mathcal{R}=\left\{(x, y) \in \mathbb{R}^{2}:-2 \leq x \leq 2,-2 \leq y \leq 2\right\}
$$

of $401 \times 401$ equidistant grid points which contains all the roots of the system. Each such point $\left(x_{0}, y_{0}\right) \in \mathcal{R}$ is assigned a color in accordance with the root at which the corresponding iterative method starting from $\left(x_{0}, y_{0}\right)$ converges. The point is marked black if either the method converges to infinity or it does not converge, with a tolerance of $10^{-8}$ in a maximum of 50 iterations. In this way, we distinguish the basins of attraction by their respective colors for different methods.

The basins of attraction, for the considered examples employing Newton's method (1.2) and the three-step Newton-like method (1.3), have been displayed in Fig. 1. It can be observed in Fig.1 that the basins of attraction generated by method (1.3) are smaller in size as compared to that generated by Newton's method. Therefore, the black points, which are considered as the bad initial points, are more in number in case of the former method. This phenomenon is observed because, the method (1.3) has order of convergence eight, in comparison to the quadratically convergent Newton's method. The figures presented in this work are performed in a 4-core 64-bit Windows machine with Intel Core i7-3770 processor using MATLAB programming language.

\section{References}

[1] Argyros, I.K., Ezquerro, J.A., Gutiérrez, J.M., Herńandez, M.A., Hilout, S., On the semilocal convergence of efficient Chebyshev-Secant-type methods, J. Comput. Appl. Math., 235(2011), 3195-3206.

[2] Argyros, I.K., George, S., Thapa, N., Mathematical Modeling for the Solution of Equations and Systems of Equations with Applications, Vol. I, Nova Publishes, NY, 2018.

[3] Argyros, I.K., George, S., Thapa, N., Mathematical Modeling for the Solution of Equations and Systems of Equations with Applications, Vol. II, Nova Publishes, NY, 2018.

[4] Argyros, I.K., Hilout, S., Weaker conditions for the convergence of Newtons method, J. Complexity, 28(2012), no. 3, 364-387.

[5] Cordero, A., Hueso, J. L., Martínez, E., Torregrosa, J.R., A modified Newton-Jarratt's composition, Numer. Algorithms, 55(2010), 87-99.

[6] Grau-Sánchez, M., Noguera, M., Amat, S., On the approximation of derivatives using divided difference operators preserving the local convergence order of iterative methods, J. Comput. Appl. Math., 237(2013), 363-372.

[7] Lotfi, T., Bakhtiari, P., Cordero, A., Mahdiani, K., Torregrosa, J.R., Some new efficient multipoint iterative methods for solving nonlinear systems of equations, Int. J. Comput. Math., 92(2015), 1921-1934.

[8] Madhu, K., Babajee, D.K.R., Jayaraman, J., An improvement to double-step Newton method and its multi-step version for solving system of nonlinear equations and its applications, Numer. Algorithms, 74(2017), 593-607.

[9] Magreñán, A.A., Argyros, I.K., Two-step Newton methods, J. Complexity, 30(2014), no. $4,533-553$. 

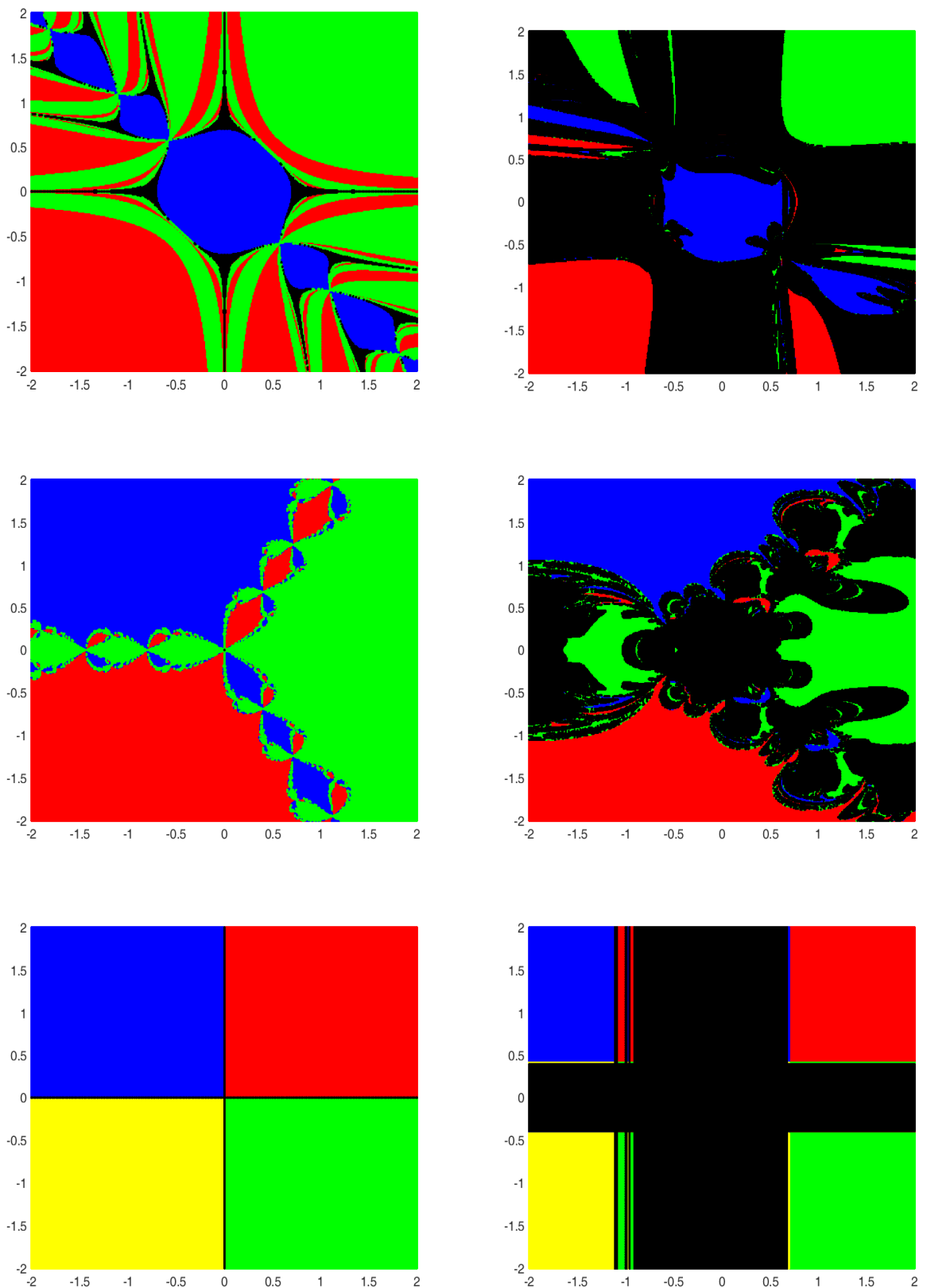

Figure 1. Basins of attraction of Example 4.1, 4.2, 4.3 using Newton's method (1.2) (1st col., top to bottom) and using the Newtontype method (1.3) (2nd col., top to bottom). 
[10] Magreñán, A.A., Argyros, I.K., Improved convergence analysis for Newton-like methods, Numer. Algorithms, 71(2016), no. 4, 811-826.

[11] Magreñán, A.A., Cordero, A., Gutiérrez, J.M., Torregrosa, J.R., Real qualitative behavior of a fourth-order family of iterative methods by using the convergence plane, Math. Comput. Simulation, 105(2014), 49-61.

[12] Narang, M., Bhatia, S., Alshomrani, A.S., Kanwar, V., General efficient class of Steffensen type methods with memory for solving systems of nonlinear equations, J. Comput. Appl. Math., 352(2019), 23-39.

[13] Osrowski, A.M., Solution of Equations and Systems of Equations, Academic Press, New York, 1960.

[14] Potra, F.A., Pták, V., Nondiscrete Induction and Iterative Processes, vol. 103, Pitman Advanced Publishing Program, 1984.

[15] Rheinboldt, W.C., An adaptive continuation process for solving systems of nonlinear equations, in: Mathematical models and numerical methods (A.N. Tikhonov et al. eds.), Banach Center, Warsaw Poland, pub. 3, 1977, 129-142.

[16] Sharma, J.R., Argyros, I.K., Kumar, S., Ball convergence of an efficient eighth order iterative method under weak conditions, Mathematics, 6(2018), no. 11, 260, https://doi.org/10.3390/math6110260.

[17] Sharma, J.R., Arora, H., Improved Newton-like methods for solving systems of nonlinear equations, SeMA J., 74(2017), no. 2, 147-163.

[18] Sharma, J.R., Arora, H., A novel derivative free algorithm with seventh order convergence for solving systems of nonlinear equations, Numer. Algorithms, 67(2014), 917-933.

[19] Traub, J.F., Iterative Methods for the Solution of Equations, AMS Chelsea Publishing, 1982.

Ioannis K. Argyros

Department of Mathematical Sciences, Cameron University,

Lawton, OK 73505, USA

e-mail: iargyros@cameron.edu

Santhosh George

Department of Mathematical and Computational Sciences,

National Institute of Technology Karnataka, Surathkal,

India- 575025

e-mail: sgeorge@nitk.edu.in

Kedarnath Senapati

Department of Mathematical and Computational Sciences,

National Institute of Technology Karnataka, Surathkal,

India-575025

e-mail: kedar@nitk.edu.in 